\title{
NORMAL MODES OF A LATERALLY HETEROGENEOUS BODY: A ONE-DIMENSIONAL EXAMPLE
}

\author{
By Robert J. Geller* and Seth Stein
}

\begin{abstract}
Various methods, including first- and second-order perturbation theory and variational methods have been proposed for calculating the normal modes of a laterally heterogeneous earth. In this paper, we test all three of these methods for a simple one-dimensional example for which the exact solution is available: an initially homogeneous "string" in which the density and stiffness are increased in one half and decreased in the other by equal amounts. It is found that first-order perturbation theory (as commonly applied in seismology) yields only the eigenvalues and eigenfunctions for a string with the average elastic properties; secondorder perturbation theory is worse, because the eigenfunction is assumed to be the original eigenfunction plus small correction terms, but actually may be almost completely different.

The variational method (Rayleigh-Ritz), using the unperturbed modes as trial functions, succeeds in giving correct eigenvalues and eigenfunctions even for modes of high-order number. For the example problem only the variational solution correctly yields the transient solution for excitation by a point force, including correct amplitudes for waves reflected by and transmitted through the discontinuity. Our result suggests but does not demonstrate, that the variational method may be the most appropriate method for finding the normal modes of a laterally heterogeneous earth model, particularly if the transient solution is desired.
\end{abstract}

\section{INTRODUCTION}

The problem of finding the normal modes of a laterally heterogeneous earth model has been considered by Madariaga (1972), Saito (1971), and Luh (1974) using firstorder degenerate perturbation theory. Luh (1973) considered the effect of quasidegeneracy. Usami (1971) used variational methods to find some very low-order torsional modes of a laterally heterogeneous earth model. Luh (1974) suggested that the variational method might be appropriate for some low-order modes. Johnson and Smylie (1977) use a variational method with splines as trial functions to directly calculate low-order modes and undertones of a rotating and elliptical earth.

The problem of lateral heterogeneity is extremely important in terms of our knowledge of the earth's interior, particularly the structural differences between oceans and continents. Currently, the agreement between observed and theoretical eigenfrequencies for spherically symmetric earth models is nominally better than 0.1 per cent for well-constrained modes. However, on the basis of the dispersion of long-period surface waves (Dziewonski, 1970; Kanamori, 1970; Leeds et al., 1974; Okal, 1977), the difference between continental and oceanic velocity structures is on the order of 5 to 10 per cent. Thus, in view of the fact that the lateral variations are much larger than the uncertainty in experimental data, and the relatively poor fits between current models and observed long-period group velocity curves, the calculation of the normal modes of laterally heterogeneous earth models seems called for. This point was emphasized by Madariaga and Aki (1972).

* Currently also at: Department of Geophysics, Stanford University, California 94305. 
The three possible methods for calculation of the normal modes of a laterally heterogeneous earth model are first- and second-order perturbation theory and the variational method. In this paper, we test these methods for a simple one-dimensional example which includes all the features of the three-dimensional problem except the degeneracy of the unperturbed problem. This test allows a comparison between an exact solution and the approximate solutions. Such tests cannot be performed for the three-dimensional case, because closed form solutions do not exist. As we show below, the variational method is much more successful than either first- or second-order perturbation theory.

\section{Approximation Methods for Normal Mode Problems}

The variational method and first- and second-order perturbation theory are well known methods in mathematical physics, so a brief review will suffice. The equation of motion for an elastic, nongravitating, body may be written as

$$
H \Psi+\rho \omega^{2} \Psi=0
$$

where $H$ is the (Hermitian) operator for the elastic restoring force, $\rho$ is the density, $\omega$ is the eigenfrequency, and $\Psi$ is the eigenfunction. The boundary conditions are

$$
\alpha \Psi+\beta \frac{\partial \Psi}{\partial n}=0
$$

where $\alpha$ and $\beta$ are arbitrary constants which may be functions of position along the boundary.

All three approximation methods assume that we start with the exact (nondegenerate) eigenvalues and eigenfunctions for the problem

$$
H^{(0)} \Psi^{(0)}+\rho^{(0)}\left(\omega^{(0)}\right)^{2} \Psi^{(0)}=0 .
$$

For simplicity, we assume that for the boundary conditions $\alpha_{0}=\alpha$ and $\beta_{0}=\beta$ (i.e., the boundary conditions are the same for the perturbed and unperturbed problems). We denote the eigenvalues and eigenfunctions for (3) as $\omega_{n}{ }^{(0)}$ and $\Psi_{n}{ }^{(0)}$, respectively. The $\Psi_{n}{ }^{(0)}$ are orthonormalized so that $\int\left(\Psi_{m}{ }^{(0)}\right)^{*} \rho^{0}\left(\Psi_{n}{ }^{(0)}\right) d V=\delta_{m n}$.

We now consider the details of each approximation method.

First-order perturbation theory: We write the perturbed operator and density in (1) as

and

$$
H=H^{(0)}+H^{(1)}
$$

where $H^{(1)}$ and $\rho^{(1)}$ represent the difference between the perturbed and unperturbed problems. We then define matrix elements

$$
H_{m n}^{(1)}=\int\left(\Psi_{m}{ }^{(0)}\right)^{*} H^{(1)}\left(\Psi_{n}{ }^{(0)}\right) d V
$$

and

$$
\rho_{n n}^{(1)}=\int\left(\Psi_{m}{ }^{(0)}\right)^{*} \rho^{(1)}\left(\Psi_{n}{ }^{(0)}\right) d V
$$


(Note that $H_{m n}^{(1)}=\left(H_{m m}^{(1)}\right)^{*}$ and $\rho_{n m}^{(1)}=\left(\rho_{m n}^{(1)}\right)^{*}$.)

In first order perturbation theory, as commonly applied in seismology, we assume that the eigenfunction for a given mode remains the same, and calculate the "firstorder" correction to the eigenvalue, $\left(\omega^{2}\right)=\left(\omega^{(1)}\right)^{2}+\left(\omega^{(0)}\right)^{2}$.

$$
\left(\omega_{n}^{(1)}\right)^{2}=-\left(\omega_{n}{ }^{(0)}\right)^{2} \rho_{n n}^{(1)}-H_{n n}^{(1)}
$$

Second-order perturbation theory: We proceed in the same way as Merzbacher (1970), except for the extra terms due to the density perturbation. We assume that we make a small perturbation to the eigenfunction, which we find to first order

$$
\Psi_{n}=\Psi_{n}{ }^{(0)}+\sum_{m \neq n} C_{n m} \Psi_{m}{ }^{(0)}
$$

and a small second-order correction to the eigenvalue

$$
\omega^{2}=\left(\omega^{(0)}\right)^{2}+\left(\omega^{(1)}\right)^{2}+\left(\omega^{(2)}\right)^{2}
$$

When we introduce (8) and (7) into (1) we obtain, after discarding terms which are not of first order

$$
C_{n m}=\frac{H_{m n}^{(1)}+\left(\omega_{n}^{(0)}\right)^{2} \rho_{m n}^{(1)}}{\left(\omega_{n}^{(0)}\right)^{2}-\left(\omega_{m}{ }^{(0)}\right)^{2}}
$$

When we substitute $(7),(8),(9)$, and $(10)$ into (1) we find the second-order correction to the eigenfrequency

$$
\left(\omega_{n}{ }^{(2)}\right)^{2}=\sum_{m \neq n} \frac{\left|H_{m n}^{(1)}+\left(\omega_{n}{ }^{(0)}\right)^{2} \rho_{m n}^{(1)}\right|^{2}}{\left(\omega_{m}{ }^{(0)}\right)^{2}-\left(\omega_{n}{ }^{(0)}\right)^{2}}-\left(\omega_{n}{ }^{(1)}\right)^{2} \rho_{n n}^{(1)} .
$$

Finally, it is necessary to renormalize the new eigenfunction. Although the summations in (8) and (11) are in principle over all modes, in practice we use some finite subset of the modes for which (11) will converge. The normalization factor is then

$$
P=\sum_{k} \sum_{m} C_{n k}^{*} C_{n m}\left[\rho_{k m}^{(1)}+\delta_{k m}\right]
$$

and the normalized eigenfunction is

$$
\Psi_{n}=\frac{1}{\sqrt{P}}\left(\Psi_{n}^{(0)}+\sum_{m \neq n} C_{n m} \Psi_{m}{ }^{(0)}\right)
$$

Dahlen (personal communication) has pointed out the existence of a boundary perturbation term associated with the jump condition. Since our goal was to conduct a one-dimensional test of the way first-order perturbation theory is applied to the earth, and such a term is not included in the three-dimensional calculation, we have omitted this boundary term in our one-dimensional calculations.

Variational method: It should be emphasized that the variational method is based on Hamilton's principle, and thus can be justified physically ( $c f$. Morse and, Feshbach, 1953). Hamilton's principle states that for any small change in a system, the La- 
grangian, $L$, is stationary,

$$
\delta(L)=\delta(T-V)=0
$$

where $T$ is the kinetic energy and $V$ is the potential energy. We will use the eigenfunctions for (3), the unperturbed solution, as trial functions and write the eigenfunction as

$$
\Psi=\sum C_{n} \Psi_{n}^{(0)}
$$

If we insert (15) into (14) and set the partial derivatives of $L$ with respect to $C_{n}$ to zero, we obtain a matrix eigenvalue problem

$$
\operatorname{det}\left(\omega^{2} T-V\right)=0
$$

where the elements of $T$ and $V$ are

$$
\begin{aligned}
& T_{i j}=\int\left(\Psi_{i}{ }^{(0)}\right)^{*}{ }^{*} \Psi_{j}{ }^{(0)} d V=\rho_{i j}^{(1)}+\delta_{i j} \\
& V_{i j}=-\int\left(\Psi_{i}{ }^{(0)}\right)^{*} H \Psi_{j}{ }^{(0)} d V=-H_{i j}^{(1)}+\delta_{i j}\left(\omega_{j}{ }^{(0)}\right)^{2} .
\end{aligned}
$$

The eigenfrequencies are given by the eigenvalues of (16) and the eigenfunctions by (15), where the coefficients $C_{n}$ are from the eigenvectors of (16).

Because the eigenfunctions for (3) are a complete set, if we used all of the unperturbed modes in our trial function, (15), we would obtain the exact eigenfrequencies and eigenvalues for the perturbed problem. In practice we must limit ourselves to a finite set and verify "experimentally" that our set is large enough. Note that, as is well known, first-order perturbation theory is essentially equivalent to the variational method with only one trial function in (15).

\section{One-Dimensional Example}

For our comparison of the three approximation methods with the exact solution, we consider a one-dimensional "string", with variable "stiffness", $k$, and "density", $p$. The Fourier transformed equation for the string is

$$
\frac{d}{d x}\left(k(x) \frac{d \Psi(x)}{d x}\right)+\rho(x) \omega^{2} \Psi(x)=0
$$

with boundary condition

$$
\Psi(0)=\Psi(l)=0
$$

If $k=k_{0}$ and $\rho=\rho_{0}$ are constants, then the eigenfrequencies are given by $\omega_{n}{ }^{(0)}=$ $n \pi c_{0} / l$, where $c_{0}=\sqrt{k_{0} / \rho_{0}}$, and the eigenfunctions by $\Psi_{n}{ }^{(0)}=\sqrt{2 / l_{\rho_{0}}} \sin \left(\omega_{n}{ }^{\left({ }^{0}\right)} x / c_{0}\right)$.

We introduce a perturbation by increasing the density and stiffness in the right 
part of the string and decreasing it by the same amount in the left part. We set

and

$$
k(x)= \begin{cases}k_{0}-(\delta k) \equiv k_{1} & 0<x<a \\ k_{0}+(\delta k) \equiv k_{2} & a<x<l\end{cases}
$$

$$
\rho(x)= \begin{cases}\rho_{0}-(\delta \rho) \equiv \rho_{1} & 0<x<a \\ \rho_{0}+(\delta \rho) \equiv \rho_{2} & a<x<l\end{cases}
$$

Exact solution: This is an extremely straightforward problem which is almost identical to the common problem of a "string" with variable density only (cf. Lapwood, 1975; Anderssen and Cleary, 1974). The solution is

$$
\Psi(x)= \begin{cases}\sin \frac{\omega x}{c_{1}} & 0<x<a \\ A \sin \frac{\omega x}{c_{2}}+B \cos \frac{\omega x}{c_{2}} & a<x<l\end{cases}
$$

where $\omega$ is picked so that $\Psi(l)=0$ and $c_{1}=\sqrt{k_{1} / \rho_{1}}$ while $c_{2}=\sqrt{k_{2} / \rho_{2}} . A$ and $B$ are found by requiring continuity of displacement and traction across $x=a$.

$$
\begin{aligned}
& A=\left(\sin \frac{\omega a}{c_{1}}\right)\left(\sin \frac{\omega a}{c_{2}}\right)+\left(\cos \frac{\omega a}{c_{1}}\right)\left(\cos \frac{\omega a}{c_{2}}\right) \cdot \frac{\rho_{1} c_{1}}{\rho_{2} c_{2}} \\
& B=\left(\cos \frac{\omega a}{c_{2}}\right)\left(\sin \frac{\omega a}{c_{1}}\right)-\left(\cos \frac{\omega a}{c_{1}}\right)\left(\sin \frac{\omega a}{c_{2}}\right) \cdot \frac{\rho_{1} c_{1}}{\rho_{2} c_{2}} .
\end{aligned}
$$

For the numerical examples in this paper we will consider a string which is asymmetric about the middle; that is, we set $a=l / 2$. For this case the secular equation simplifies to

$$
\Psi(l)=\sin \frac{\omega l}{2 c_{1}} \cos \frac{\omega l}{2 c_{2}}+\frac{\rho_{1} c_{1}}{\rho_{2} c_{2}} \cos \frac{\omega l}{2 c_{1}} \sin \frac{\omega l}{2 c_{2}}=0
$$

which may be written in a slight generalization of Lapwood's (1975) result as

$$
\left(\frac{k_{1}}{c_{1}}+\frac{k_{2}}{c_{2}}\right) \sin \left(\frac{\omega l}{2}\left(\frac{1}{c_{1}}+\frac{1}{c_{2}}\right)\right)=-\left(\frac{k_{1}}{c_{1}}-\frac{k_{2}}{c_{2}}\right) \sin \left(\frac{\omega l}{2}\left(\frac{1}{c_{1}}-\frac{1}{c_{2}}\right)\right) .
$$

Equation (25) demonstrates the existence of a solotone effect (Lapwood, 1975; McNabb et al., 1976); that is, no matter how large $\omega$ becomes the eigenvalues will oscillate around an asymptote rather than converging on it. This asymptote is given by $\omega=2 n \pi / l\left(1 / c_{1}+1 / c_{2}\right)$. Thus the asymptote is $n \pi / l$ divided by the average slowness. The solotone effect is closely related to the generation of reflected phases whenever a wave crosses the boundary at $x=l / 2$ (cf. Wang et al., 1977; Anderssen, 1977).

The normalization of (22) for $a=l / 2$ is given by

$$
\begin{aligned}
Q \equiv \int_{0}^{l} \rho \Psi^{2} d x=\rho_{1}\left[\frac{l}{4}-\frac{c_{1}}{4 \omega} \sin \frac{\omega l}{c_{1}}\right] & +\rho_{2}\left[\frac{l}{4}\left(A^{2}+B^{2}\right)\right. \\
+ & \frac{c_{2}}{4 \omega}\left(B^{2}-A^{2}\right)\left(\left(\sin \frac{2 \omega l}{c_{2}}\right)-\left(\sin \frac{\omega l}{c_{2}}\right)\right) \\
& \left.-\frac{c_{2}}{2 \omega} A B\left(\left(\cos \frac{2 \omega l}{c_{2}}\right)-\left(\cos \frac{\omega l}{c_{2}}\right)\right)\right]
\end{aligned}
$$


and the normalized eigenfunctions are

$$
\Psi(x)= \begin{cases}\left(\sin \frac{\omega x}{c_{1}}\right) / \sqrt{Q} & 0<x<l / 2 \\ \left(A \sin \frac{\omega x}{c_{2}}+B \cos \frac{\omega x}{c_{2}}\right) / \sqrt{Q} & l / 2<x<l .\end{cases}
$$

First-order perturbation theory: We find that the diagonal matrix elements defined in (6), $\rho_{n n}^{(1)}$ and $H_{n n}^{(1)}$ are zero. Thus, to first order, there is no change in the average velocity along the string. (For the three-dimensional case, Jordan (1977) has shown that the eigenvalues obtained from first-order perturbation theory for a laterally homogeneous earth correspond to the average phase velocity along a particular great circle path; the same result can be shown for the initially homogeneous string.) Thus the first-order correction to the eigenvalue is zero

$$
H_{n n}^{(1)}=\rho_{n n}^{(1)}=0 .
$$

Second-order perturbation theory: For the perturbations (20) and (21) we obtain off-diagonal matrix elements

$$
\begin{aligned}
& \boldsymbol{\rho}_{m n}^{(1)}=\frac{2(\delta \rho)}{\rho_{0} \pi}\left[\frac{\sin \frac{(m+n) \pi}{2}}{m+n}-\frac{\sin \frac{(m-n) \pi}{2}}{m-n}\right] \\
& H_{m n}^{(1)}=\frac{2(\delta k) m n \pi}{l^{2} \rho_{0}}\left[\frac{\sin \frac{(m+n) \pi}{2}}{m+n}+\frac{\sin \frac{(m-n) \pi}{2}}{m-n}\right]
\end{aligned}
$$

$(m \neq n)$. We then use the matrix elements in (10) and (11) to find the eigenfunctions and eigenvalues, respectively.

Variational method: We use the off-diagonal matrix elements from (29) and diagonal elements from (28) to obtain the elements of the variational matrices, (17). We then must solve the matrix eigenvalue problem

$$
V X=\omega^{2} T X .
$$

Before we can actually solve (30), we must decide which trial functions to use. We decided, because of computational considerations, to use 41 trial functions. In finding modes with $1 \leqq n \leqq 21$, we used $\Psi_{1}{ }^{(0)}$ through $\Psi_{41}^{(0)}$ as trial functions; for modes with $n>21$ we used $\Psi_{n-20}^{(0)}$ through $\Psi_{n+20}^{(0)}$.

We chose to find the eigenvalue, as well as the eigenfunction, by inverse iteration (we replace $\omega^{2}$ by $\lambda$ for convenience). Starting with an initial guess for an eigenfunction, $X^{(0)}$, the Rayleigh quotient is used to estimate the eigenvalue

$$
\lambda^{(0)}=\frac{X^{T} V X}{\bar{X}^{T} T \bar{X}}
$$

We then use the guess for the eigenvalue, $\lambda^{(i)}$ to find a new guess for the eigenvector, $\underline{X}^{(i+1)}$ by solving the inhomogeneous linear equation

$$
\left(V-\lambda^{(i)} T\right) X^{(i+1)}=T X^{(i)}
$$


Wilkinson (1965) shows that the convergence toward the desired eigenvector is extremely rapid, if $\lambda^{(i)}$ is a good estimate of the eigenvalue. Following Wiggins (1976) we then use the new estimate of the eigenvector to obtain the next eigenvalue estimate from the Rayleigh quotient. We continue with this procedure until convergence is obtained; in practice, only two iterations of (32) were ever necessary. After convergence is obtained, we normalize $\boldsymbol{X}$ by using the denominator of the Rayleigh quotient

$$
R=X^{T} T X
$$

in the normalized eigenfunction

$$
\Psi=\sum X_{i} \Psi_{i}^{(0)} / \sqrt{R}
$$

In order for this method to succeed, we need good initial guesses for the eigenvector. These guesses are obtained in the following way. In finding the gravest mode we set $X^{(0)}=(1,0,0,0, \cdots)^{T}$. Thereafter, we used the eigenfunction $Y$ for the previous mode "shifted up" by one position as the initial guess

$$
X^{(0)}=\left(0, Y_{1}, Y_{2}, Y_{3}, \cdots\right)^{T} .
$$

It can be shown that this yields a very good initial guess. In our discussion of the variational method, the fact that we are using only a finite set of trial functions is implicit in our subscripts; we do not explicity include this in expressions such as (34).

\section{Numerical Example}

We present a comparison of the exact, variational, first-order perturbation and second-order perturbation methods for a specific example. We set $k_{1}=0.9, k_{2}=1.1$, $\rho_{1}=0.4, \rho_{2}=0.6$, and $l=20$. Thus, $c_{1}=1.5, c_{2}=1.35, k_{0}=0, \rho_{0}=0.5, \delta k=0.1$, and $\delta \rho=0.1$. The eigenfrequencies for each method (plotted as "reduced" frequencies, $\omega-\omega^{(0)}$ ) are given in Figure 1. The eigenfrequencies for the exact solution are plotted as plus signs.

The eigenfrequency for first-order perturbation theory is the same as the unperturbed eigenfrequency, so the first-order perturbation theory does not reflect the change in elastic properties at all. Woodhouse (1976) has also shown that Rayleigh's principle may be inappropriate for problems of this type. The second-order perturbation eigenfrequencies diverge very rapidly from the true eigenfrequency although they oscillate wildly about the correct asymptote. This is not because the second-order series did not converge. It did-to the wrong answer. However, if additional terms of higher order were included in the perturbation series, it would presumably converge to the correct result eventually.

The agreement between the exact and variational solutions is excellent. The solotone effect and the asymptotic behavior discussed previously can be clearly seen. Only for very high $n$ does the agreement start to deteriorate at all, and this could easily be corrected by using more trial functions.

Besides comparing the eigenfrequencies for different methods, we compare the expansion coefficients for the eigenfunctions. We expand the exact solution for the eigenfunction, (22) and (23), in terms of the unperturbed eigenfunctions

$$
\Psi(x)=\sum_{n} C_{n} \Psi_{n}^{\left({ }^{(0)}\right.}(x)
$$


where

$$
C_{n}=\int_{0}^{l} \rho_{0} \Psi_{n}^{(0)}(x) \Psi(x) d x
$$

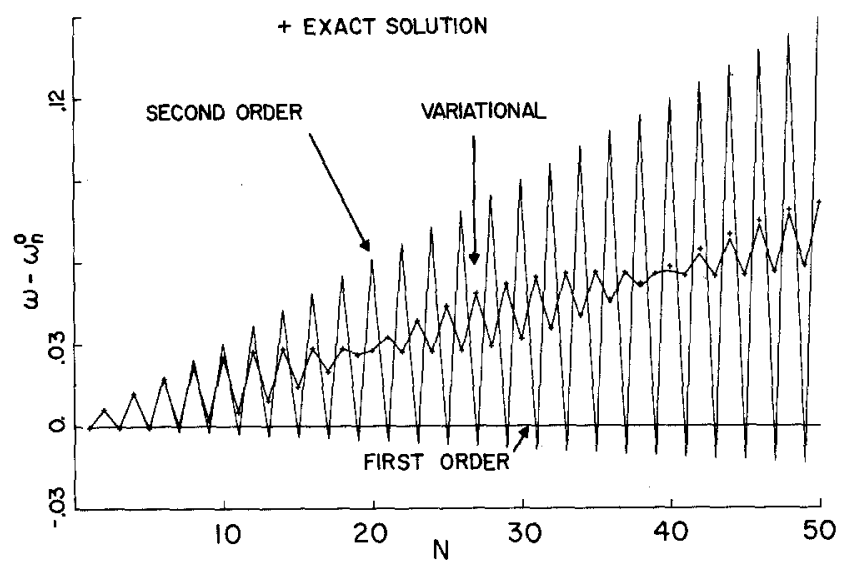

Fig. 1. Comparison of eigenfrequencies for exact solution, variational method, and first- and second-order perturbation theory. $\omega_{n}{ }^{(0)}$ is subtracted from all eigenvalues for ease of presentation. The variational solution agrees excellently with the exact solution, while the secondorder perturbation solution oscillates wildly around the correct asymptote. First-order perturbation yields eigenvalues which are identical to those for the unperturbed problem. To put this figure in perspective, the lowest eigenvalue is $\omega_{1}=0.2219$, so the difference between $\omega_{50}$ and $\omega_{50}^{(0)}$ is about $\frac{1}{3}$ of $\omega_{1}$.

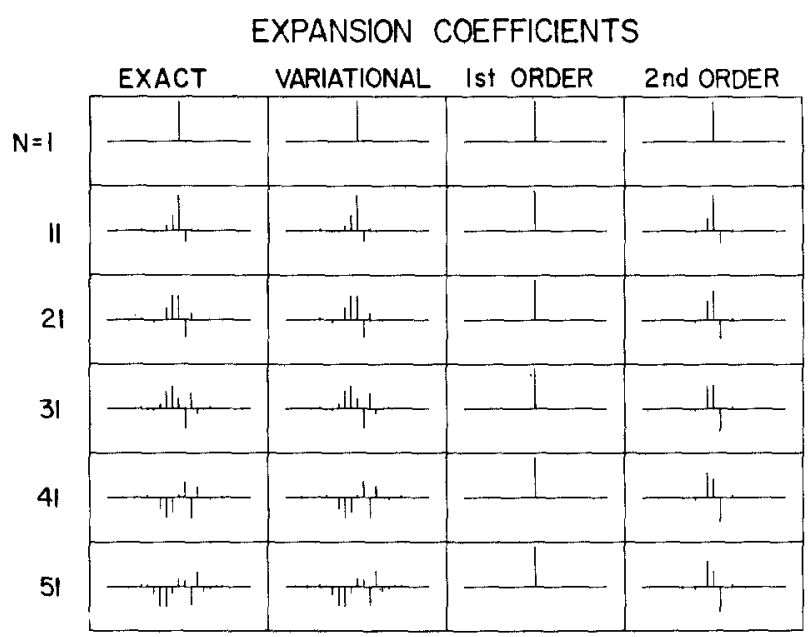

Frg. 2. Comparison of expansion coefficients for exact solution, variational method, and first- and second-order perturbation theory, for modes of order $N$. In each case the coefficient for the $N$ th mode is in the center.

Evaluation of the integral in (36) is straightforward and need not be given here. The expansion coefficients for the variational and perturbation methods are obtained directly from the normalized solutions.

The expansion coefficients for all four methods are shown in Figure 2 for modes $n=1,11,21,31,41$, and 51 . The normalized coefficient of the $n$th unperturbed mode is plotted in the center of each box. Note that in Figures 2 and 3 "first" and "second" 
order refer to the order of the eigenvalue, and the corresponding eigenfunctions are "zeroeth" or "first" order. For all methods except the trivial case of first-order perturbation theory, the extent to which the eigenfunction is composed of other modes besides $\Psi_{n}{ }^{(0)}$ increases with increasing $n$. All three approximate methods yield acceptable results for $n=1$, for which the eigenfunction is basically unchanged from the unperturbed case. Even for the relatively low-order case of $n=11$, certain distinctive patterns are established. The expansion coefficients for the exact solution show that the eigenfunction is not composed of the original, unperturbed, eigenfunction plus some very small amounts of the other original eigenfunctions. The amplitudes of the coefficients of $\Psi_{10}^{(0)}$ and $\Psi_{12}^{(0)}$ are about 40 per cent of the coefficient of $\Psi_{11}^{(0)}$, so it is not appropriate to regard these as 'small' correction terms. Thus, perturbation theory is inapplicable. Comparing the second-order perturbation solution to the exact solution, we observe that the relative sizes of the coefficients which are non-zero are roughly correct. However, because of the form of the coefficients, (10) and the matrix elements, (28) and (29), we see that, as we move away from the central mode in either direction, every other coefficient is zero. Thus, although the relative sizes of the coefficients which are nonzero are about right, the omission of half of the terms which should be included causes serious errors in both the eigenvalues (see Figure 1) and eigenfunctions.

As $n$ increases, the number of unperturbed modes which contribute significantly to the perturbed solution increases substantially, as is shown by the examples for $n=41$ or $n=51$. For these high orders, even the relative size of the second-order expansion coefficients is completely wrong. We see an interesting effect for the coefficient of $\Psi_{n}{ }^{(0)}$. For $n=1$ and 11, this is the dominant coefficient, for $n=21$ it is essentially equal to the dominant coefficient, and for $31 \leqq n \leqq 51$ it is smaller in magnitude than the most significant coefficients. The effect is particularly striking for $n=41$, for which $C_{41}$ is less than 20 per cent of the largest coefficient. This example demonstrates that it is easily possible for the original eigenfunction to make up a negligible part of the mode of the perturbed problem with the same order number. This is a fundamental contradiction of the assumption of "small" corrections inherent in perturbation theory.

\section{Transient Solution}

We consider the problem of finding the fundamental solution for a force which has a $\delta$-function dependence in space and time. After taking the Fourier transform of the equation of motion we have

$$
H \Psi+\rho \omega^{2} \Psi=-\delta\left(x-x_{0}\right)
$$

we assume a particular solution of the form $\Psi=\sum D_{n} \Psi_{n}$, where the $D_{n}$ are unknown coefficients and the $\Psi_{n}$ are orthonormalized eigenfunctions. Multiplying by $\Psi_{m}$, and integrating from 0 to $l$, we find

$$
D_{m}=-\frac{\Psi_{m}\left(x_{0}\right)}{\left(\omega^{2}-\omega_{m}^{2}\right)}
$$

Then, using (38) and inverting the Fourier transform we find that

$$
\Psi(x, t)=\sum_{n}-\Psi_{n}\left(x_{0}\right) \Psi_{n}(x) \frac{\sin \omega_{n} t}{\omega_{n}}
$$

If, instead of $\delta(t)$ time dependence, the time dependence of $(39)$ is $d f d t(\delta(t))_{*} \exp$ 
$\left(-t^{2} / \tau_{0}{ }^{2}\right)$, (where $\tau_{0}$ is the constant "rise time"), we get basically Gaussian pulses traveling along the string

$$
\Psi(x, t)=\sum_{n}-\Psi_{n}\left(x_{0}\right) \Psi_{n}(x) \exp \left(-\left(\omega_{n} \tau_{0}\right)^{2}\right) \cos \omega_{n} t
$$

We calculated the excitation for the exact solution using (40) and for the three approximate solutions for the case of $x_{0}=5$ and $\tau_{0}=0.075$. For each method, "snapshots" of the displacement along the string are shown in Figure 3 for times from $t=1$ through $t=50$. In each calculation, 200 modes are used. Several notable features appear readily from the comparison between methods. We see that the first-order perturbation solution does not contain any of the reflected phases from the boundary in the middle of the string, and the main pulses eventually become slightly out of phase from those for the exact solution. The solution for second-order perturbation theory, with inaccurate eigenfrequencies (Figure 1) and eigenfunctions (Figure 2), rapidly degenerates to a completely incorrect result. For example, even for the snapshot at $t=1$ the two main pulses are not symmetric and small noncausal phases appear in the right half of the string.

The agreement between the exact solution and the variational solution is excellent. Even for $t=50$, the snapshots agree pulse for pulse. From $t=1$ through $t=3$ the pulses spread away from $x_{0}=5$, the point at which the string was plucked, as they would on a completely uniform and infinite string. The effect of the boundary occurs at $t=4$. The pulse traveling on the left has been reflected by the rigid boundary and now travels to the right (away from the boundary) with the negative of its previous amplitude. Meanwhile, the rightmost pulse has passed through the first-order discontinuity at $x=10$. The reflection and transmission coefficients for the wave traveling to the right are

$$
\begin{aligned}
& R=\frac{\rho_{1} c_{1}-\rho_{2} c_{2}}{\rho_{1} c_{1}+\rho_{2} c_{2}} \\
& T=\frac{2 \rho_{1} c_{1}}{\rho_{1} c_{1}+\rho_{2} c_{2}} .
\end{aligned}
$$

For our problem, $R=-0.15$ and $T=0.85$. (For waves traveling to the left, $R=$ 0.15 and $T=1.15$.) The reflection and transmission coefficients for both the variational and exact solutions agree with these theoretical values. Note that the amplitudes of the main pulses do not decay appreciably, because the product of the transmission coefficient for passage from left-to-right times the coefficient for right-to-left transmission is $(0.85)(1.15)=0.98$. Thus the reflected pulses, although having significant amplitudes themselves, cause only a slight decrease in the size of the main pulses.

The velocity in the left half is 1.5 and the pulse which was reflected from the internal boundary has traveled 15 units when it hits the left-hand boundary at $t=10$. Since the travel time for the center of the pulse is exactly 10 units, the leading half of the pulse (which was reflected with a reflection coefficient of -1 ) exactly cancels out the trailing half, producing a net displacement of zero. The fact that the solution, as plotted, is zero, demonstrates the accuracy of our variational solution.

With increasing time, the wave forms become more and more complex as additional reflected pulses are created. (In fact there are even small secondary reflected pulses caused by passage of primary reflected pulses through the internal discontinuity!) 
Although, formally, the normal mode solution must produce all of the pulses predicted by optics, it is nonetheless extremely gratifying to observe that this actually occurs.

It is worth noting that this discussion is for an extreme case, that of a step dis-

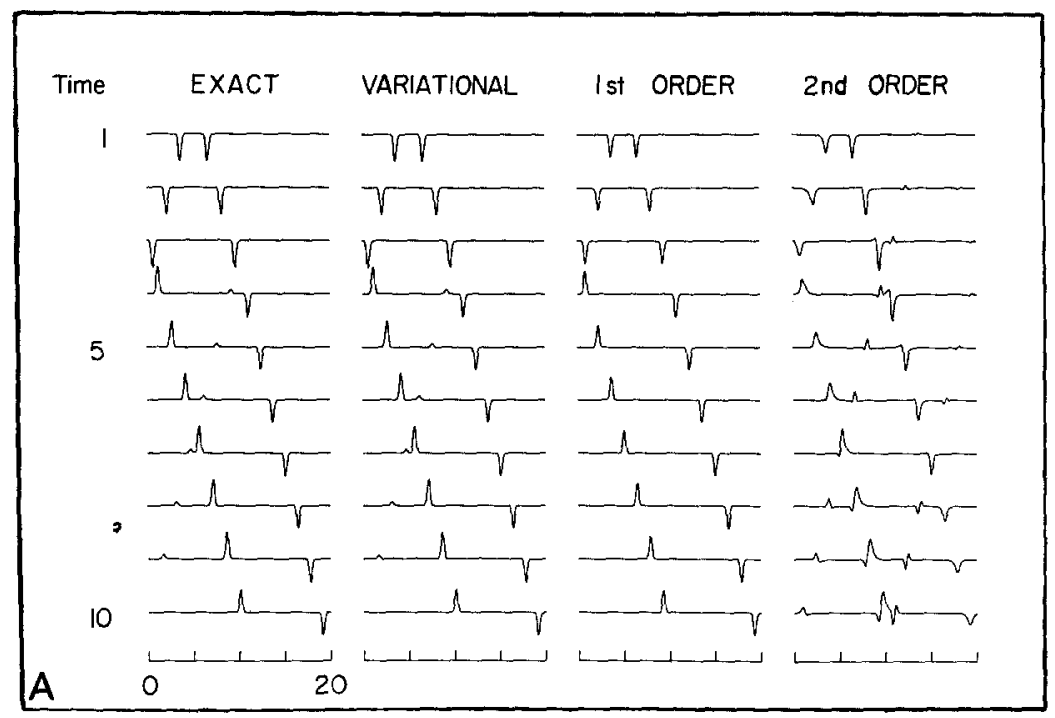

FIG. 3a. Comparison of displacement "snapshots" for exact solution, variational method and first- and second-order perturbation theory for $1 \leqq t \leqq 10 ; 200$ modes are summed in each case and all figures have the same amplitude scale.

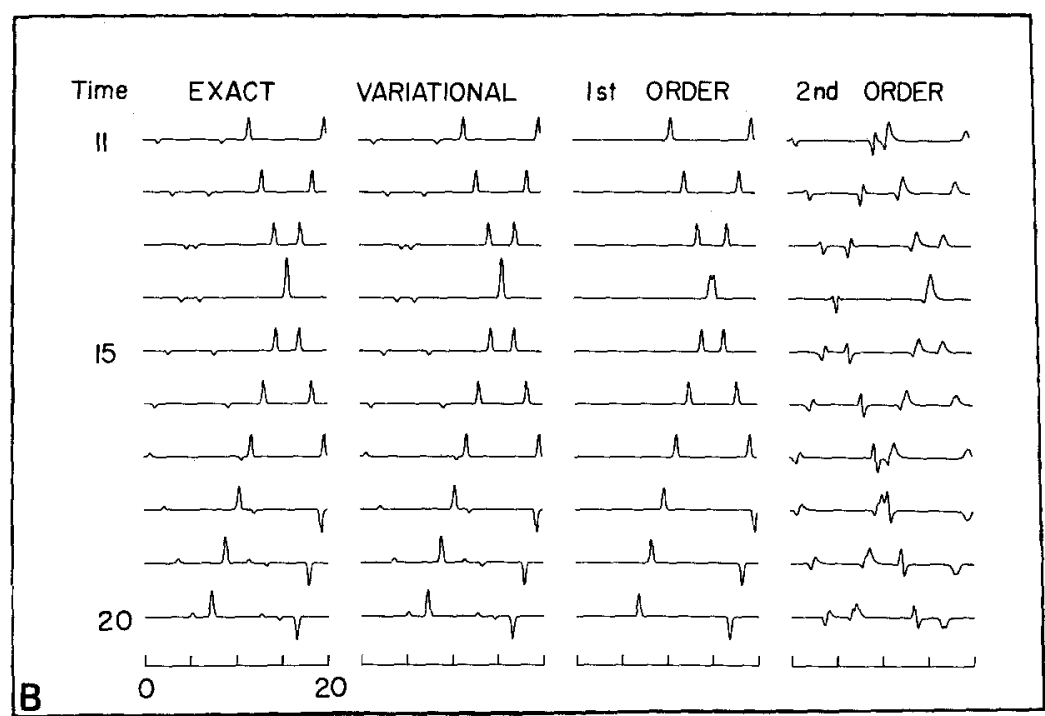

Fig. 3b. Comparison of displacement "snapshots" for exact solution variational method and first-and second-order perturbation theory for $11 \leqq t \leqq 20$.

continuity. It is possible that perturbation theory would be more successful for a more gradual transition zone.

\section{Discussion}

We have presented clear and compelling evidence that of the variational, firstorder perturbation and second-order perturbation theories, only the variational 
method yields accurate solutions for a one-dimensional lateral heterogeneity problem. Our result suggests, but does not demonstrate, that the variational method, using

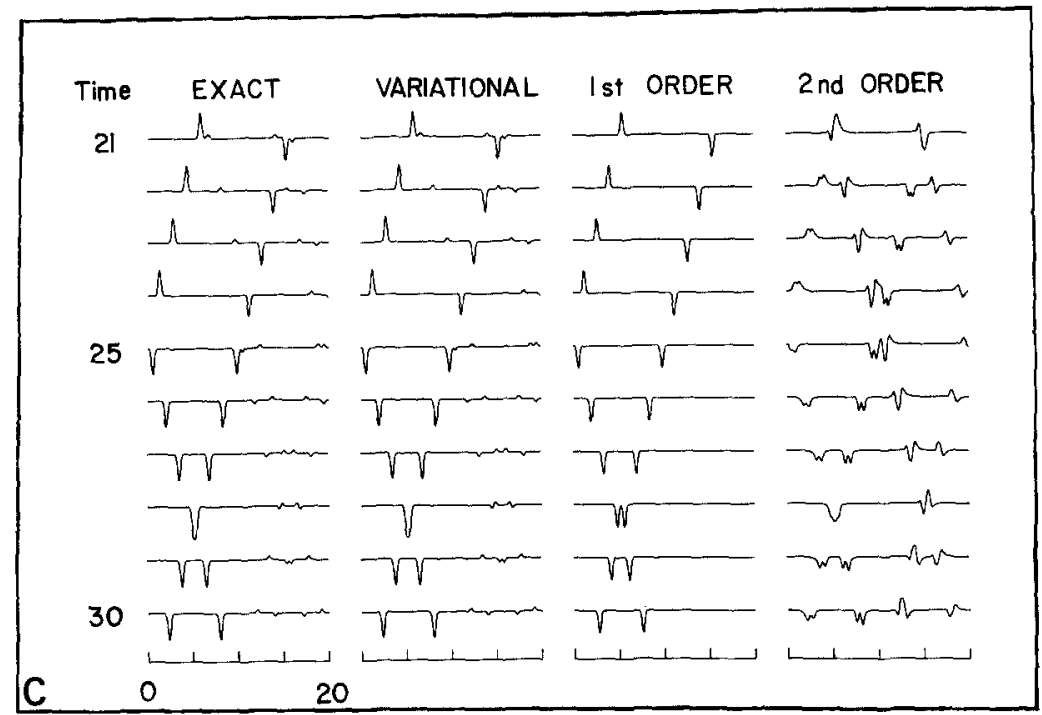

Fra. 3c. Comparison of displacement "snapshots" for exact solution, variational method and first- and second-order perturbation theory for $21 \leqq t \leqq 30$.

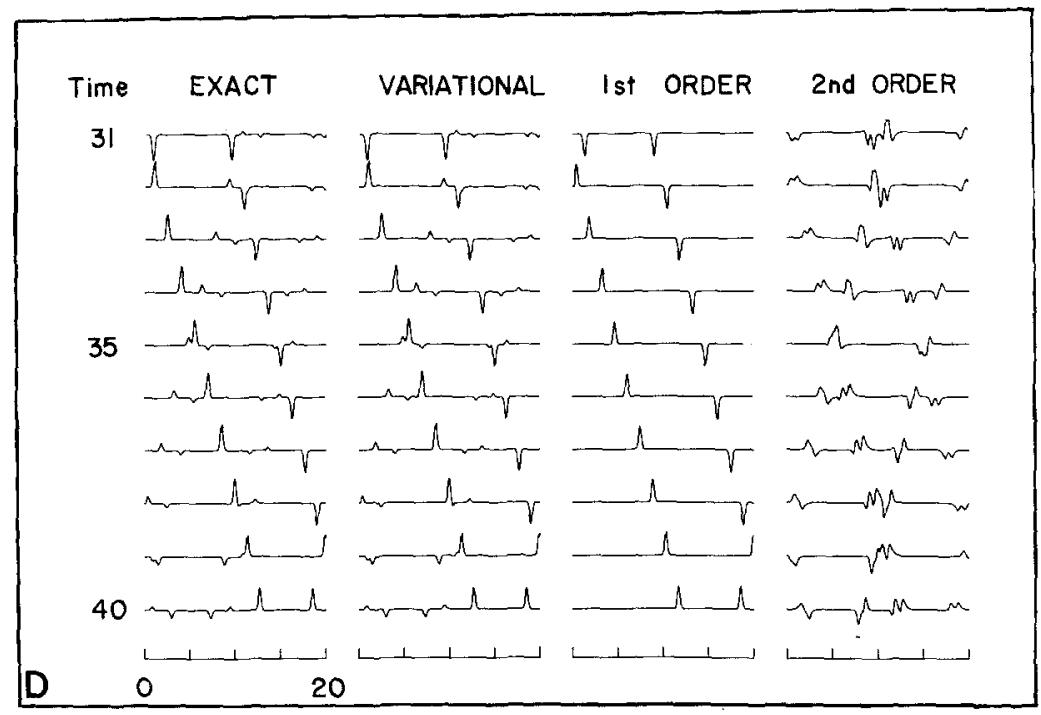

Fig. 3d. Comparison of displacement "snapshots" for exact solution, variational method and first- and second-order perturbation theory for $31 \leqq t \leqq 40$.

the unperturbed eigenfunctions as trial functions, should be strongly preferred for the three-dimensional lateral heterogeneity problem in the Earth. We now are testing this suggestion.

Figure 3 is a schematic picture of what might happen to long-period surface waves as they make multiple circuits about the Earth. Initially the wave forms are very simple, but become more and more complex as the waves propagate across discon- 


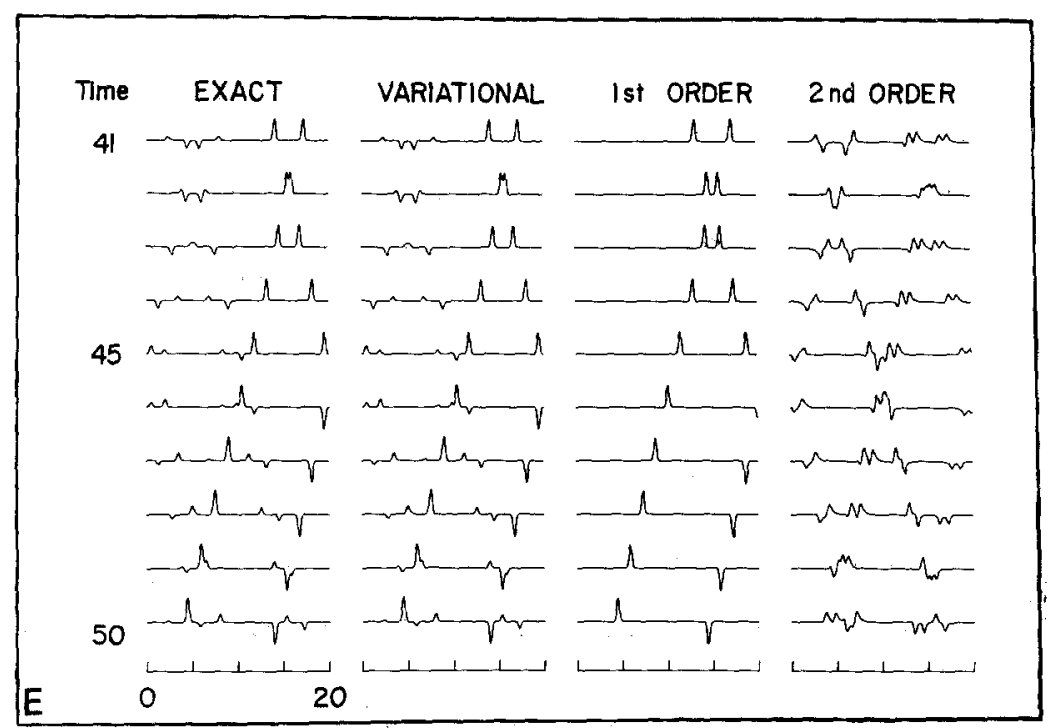

FIG. 3e. Comparison of displacement "snapshots" for exact solution, variational method and first- and second-order perturbation theory for $41 \leqq t \leqq 50$.

tinuous boundaries. Perhaps very similar phenomena occur as waves on the Earth cross continent-ocean boundaries. If so, then the variational calculation of the modes of a laterally heterogeneous Earth may help shed light on the observed wave forms, and on the nature of the Earth's lateral heterogeneity.

\section{ACKNOWLEDGMENTS}

We thank Jon Claerbout, Yoshio Fukao, Hiroo Kanamori, and Emile Okal for helpful discussions. Gene Golub, Herb Keller, and Dan Kosloff gave us useful advice on the matrix eigenvalue problem. We also thank Tony Dablen, Raul Madariaga, and Robert Anderssen for constructive comments on an earlier draft. This research was supported by the National Science Foundation under Grants EAR76-14262 and EAR 77-14675. Seth Stein was supported by a Fellowship from the Fannie and John Hertz Foundation.

\section{REFERENCES}

Anderssen, R. S. and J. R. Cleary (1974). Asymptotic structure in torsional free oscillations of the earth-I, overtone structure, Geophys. J. 39, 241-268.

Anderssen, R. S. (1977). The effect of discontinuities in density and shear velocity on the asymptotic overtone structure of torsional eigenfrequencies of the earth, Geophys. J. 50, 303-310.

Dziewonski, A. M. (1970). On regional differences in dispersion of mantle Rayleigh waves, Geophys. $J .22,289-325$.

Johnson, I. M. and D. E. Smylie (1977). A variational approach to earth dynamics, Geophys. $J$. 50, 35-54.

Jordan, T. H. (1977). A procedure for estimating lateral variations from low-frequency eigenspectra data, preprint.

Kanamori, H. (1970). Velocity and Q of mantle waves, Phys. Earth Planet Interiors 2, 259-275.

Lapwood, E. R. (1975). The effect of discontinuities in density and rigidity on torsional eigenfrequencies of the earth, Geophys. $J .40,453-464$.

Leeds, A. R., E. G. Kausel, and L. Knopoff (1974). Variations of upper mantle structure under the Pacific Ocean, Science 186, 141-143.

Luh, P. C. (1973). Free oscillations of the laterally inhomogeneous earth: quasi-degenerate multiplet coupling, Geophys. J. 32, 187-202.

Luh, P. C. (1974). Normal modes of a rotating self-gravitating inhomogeneous earth, Geophys. $J .38,187-224$. 
Madariaga, R. (1972). Toroidal free oscillations of the laterally heterogeneous earth, Geophys. $J$. 217, 81-100.

Madariaga, R. and K. Aki (1972). Spectral splitting of toroidal-free oscillations due to lateral heterogeneity of the earth's structure, $J$. Geophys. Res. 77, 4421-4431.

McNabb, A., R. S. Anderssen, and E. R. Lapwood (1976). Asymptotic behavior of the eigenvalues of a Sturm-Liouville system with discontinuous coefficients, J. Math. Anal. Appl. 54, 741-751.

Merzbacher, E. (1970). Quantum Mechanics, 2nd ed., Wiley, New York, 417-420.

Morse, P. M. and H. Feshbach (1953). Methods of Theoretical Physics, Part II, McGraw-Hill, New York, pp. 10,00, 1106-1110.

Okal, E. A. (1977). The effect of intrinsic oceanic upper-mantle heterogeneity on regionalization of long period Rayleigh-wave phase velocities, Geophys. J. 49, 357-370.

Saito, M. (1971). Theory for the elastic-gravitational oscillation of a laterally heterogeneous earth, J. Phys. Earth 19, 259-270.

Usami, T. (1971). Effect of horizontal heterogeneity of the torsional oscillation of an elastic sphere, J. Phys. Earth 19, 175-180.

Wang, C., J. F. Gettrust, and J. R. Cleary (1977). Asymptotic overtone structure in eigenfrequencies of torsional normal modes of the earth, a model study, Geophys. J, 50, 289-302.

Wiggins, R. A. (1976). A fast new computational algorithm for free oscillations and surface waves, Geophys. J. 47, 135-150.

Wilkinson, J. A. (1965). The Algebraic Eigenvalue Problem, Oxford U. Press, London, pp. 321-322.

Woodhouse, J. H. (1976). On Rayleigh's principle, Geophys. J.46, 11-22.

SEISMOLOGICAL LABORATORY

California Institute of Technology

Pasadena, California 91125

Contribution No. 2969 of The Division of Geological and Planetary Sciences

Manuscript received September 2, 1977 